\title{
Compliance des patients irradiés pour le port des gouttières de fluoration : incidence sur la carie dentaire
}

\section{Patients compliance to fluorisation trays and incidence of dental caries after radiotherapy: a retrospective analysis}

MATHILDE SAVIGNAT*, ERIC LARTIGAU**, SOPHIE VI*, PHILIPPE LIBERSA*

\section{RÉSUMÉ}

Lors de la radiothérapie des voies aérodigestives supérieures, l'irradiation des glandes salivaires entraîne une hyposialie qui génère, entre autres, des conditions cariogènes dans le milieu buccal. La prévention de ces complications repose essentiellement sur le port, cinq à dix minutes chaque soir, de gouttières de fluoration thermoformées. Le but de l'étude était d'évaluer la compliance de 100 patients irradiés pour le port des gouttières, et d'étudier l'incidence de ce port sur la carie dentaire.

Au total, $48 \%$ des patients n'ont jamais porté leurs gouttières et l'augmentation de l'incidence des caries est liée de façon significative (chi-deux, $p<10^{-6}$ ) au non-port des gouttières. L'étude montre à nouveau l'importance indéniable de la fluorothérapie dans le cadre des complications liées à la radiothérapie. Cependant, il y a lieu de s'interroger sur les raisons du faible pourcentage de patients respectant le protocole de fluorothérapie, et donc d'améliorer la prise en charge des patients irradiés. Med Buccale Chir Buccale 2007; 13 : 77-81.

mots clés: gouttières, fluor topique, radiothérapie

\section{SUMMARY}

Radiotherapy plays an important role in the treatment of head and neck cancers. The irradiation of salivary glands results in a decrease of salivary secretion. These cariogenic conditions can be lowered by the use of thermoformed fluorisation trays used five to ten minutes every evening. The aim of this retrospective study was to evaluate the compliance of 100 head and neck cancer patients to the use of fluorisation trays once daily and to determine how this compliance impacted on the risk of caries.

The study showed that $48 \%$ of the patient never used the fluorisation trays and that the mean number of caries increased significantly with the non-compliance degree. These results demonstrate that the dentist can play a key role on the post-radiotherapy complications and patients quality of life. Med Buccale Chir Buccale 2007; 13: 77-81.

key words: trays, topical fluorine, radiation therapy

\footnotetext{
* Faculté de Chirurgie dentaire, Université de Lille II, place de Verdun, 59000 Lille

${ }^{* *}$ Centre Oscar Lambret, 3 rue F. Combemale, 59000 Lille

Demande de tirés à part:

Mathilde Savignat Faculté de Chirurgie dentaire place de Verdun 59000 Lille mathilde.savignat@univ-lille2.fr
} 
médecine buccale chirurgie buccale

VOL. $13, \mathrm{~N}^{\circ} 2$ 2007

page 78
Les cancers des voies aérodigestives supérieures (VADS) ont une prévalence élevée en France, et la mortalité pour ces cancers est la plus élevée au monde avec 9,8 décès pour 100000 habitants par an [1]. Depuis 1980, l'incidence des cancers des VADS a diminué de $22 \%$ chez les hommes, mais augmenté de $36 \%$ chez les femmes. II existe également des disparités entre les différents départements, et le taux de mortalité dans le Nord et le Pas-de-Calais est 1,7 fois plus élevé que le taux national [2]. La radiothérapie joue un rôle essentiel dans le traitement des cancers des VADS. Dans la région cervico-faciale, la radiothérapie induit une irradiation des glandes salivaires et, par conséquent, une diminution de la sécrétion salivaire. La sévérité de l'atteinte des glandes salivaires est directement liée à la dose d'irradiation, au fractionnement de la radiothérapie, aux champs d'irradiation et donc au volume de glandes salivaires irradié. Histologiquement, le parenchyme salivaire montre, lorsqu'il est soumis à une radiothérapie, une inflammation aiguë initiale suivie d'une fibrose et d'une atrophie [3]. L'hyposialie consécutive à la radiothérapie génère des conditions cariogènes dans la cavité buccale : des changements quantitatifs et qualitatifs de la flore buccale, une diminution du $\mathrm{pH}$, et une perte des actions mécaniques et physico-chimiques de la salive sont observés. Chez les patients présentant une hyposialie induite par la radiothérapie, la concentration microbienne buccale totale reste proche des valeurs normales, mais l'équilibre entre microorganismes cariogènes et non-cariogènes est modifié et on observe une prédominance des microorganismes cariogènes. Une augmentation du nombre des S. mutans, Lactobacillus et Candida, concomitante à une diminution des $S$. sanguis, Neisseria et Fusobacterium, a été démontrée ${ }^{[4]}$. Ces changements de la flore sont expliqués en partie par la modification des composants protéiques de la salive induite par la radiothérapie [5].

L'hyposialie postradique se traduit cliniquement par une mucite, des caries et des candidoses fréquentes, conditions qui peuvent mener à des phénomènes douloureux, une modification de la prise alimentaire, des problèmes esthétiques et des ostéoradionécroses ${ }^{[6-8]}$. Les caries postradiques sont caractérisées par une localisation inhabituelle au collet des dents et par une grande rapidité d'évolution. Tous les sites deviennent vulnérables à la carie, et des lésions franches peuvent être observées en trois mois [9]. Les effets bénéfiques des fluorures sur les tissus minéralisés sont maintenant bien connus. Les fluorures diminuent la déminéralisation induite par la colonisation de S.mutans et inhibent le métabolisme bactérien [10,11]. L'application de fluor topique au moyen de gouttières thermoformées portées cinq à dix minutes chaque soir permet la prévention des caries postradiques. Des recommandations telles qu'une hygiène bucco-dentaire rigoureuse, une limitation de l'apport alimentaire cariogène et l'utilisation de salive artificielle favorisent également cette prévention. La fluorothérapie est un traitement à vie, qui nécessite des visites de contrôle régulières chez le chirurgien dentiste. Lors de ces visites, le chirurgien dentiste devra s'assurer du port quotidien des gouttières de fluoration et motiver le patient quand cela apparaît nécessaire.

Le but de l'étude était d'évaluer le degré de compliance des patients ayant subi une radiothérapie dans la région cervico-faciale au port quotidien des gouttières de fluoration. Les effets de la compliance sur le risque carieux ont également été déterminés chez ces patients.

\section{MALADES ET METHODES}

La cohorte comprenait 100 patients pris en charge dans un service de radiothérapie du Nord Pasde-Calais sur une période de 17 ans (1986-2002). L'examinateur a expliqué l'étude à chacun des participants, qui a lu et signé un formulaire de consentement éclairé. Les patients recrutés étaient majeurs, devaient avoir subi une radiothérapie avec les glandes salivaires principales comprises dans les champs d'irradiation. Les patients édentés ont été exclus de l'étude.

Avant le début de la radiothérapie, les foyers infectieux avaient été éliminés, puis l'âge, le sexe, la localisation tumorale et l'état dentaire initial (nombre de dents) ont été relevés. Les gouttières de fluoration avaient été réalisées et chaque 
patient avait reçu les explications quant à l'application quotidienne, pendant dix minutes chaque soir, du gel fluoré au moyen des gouttières. Ils avaient été informés de l'importance du port des gouttières pendant mais, également à vie, après la radiothérapie, à moins que la sécrétion salivaire ne revienne à la normale. Après la radiothérapie, la dose totale d'irradiation a été relevée et un contrôle dentaire réalisé de façon à détecter le nombre de caries après traitement. Sous la forme d'un questionnaire, les patients devaient informer l'examinateur du port des gouttières tous les jours, une à quatre fois par semaine ou jamais. La description statistique de la cohorte a utilisé les méthodes classiques de calcul de moyenne et d'écart-type. Les données catégorielles, exprimées en pourcentage, ont été comparées à l'aide du test du chi-deux, avec une correction de Yates quand cela était nécessaire (petits effectifs). Les comparaisons de moyennes ont été réalisées par analyse de variance et calcul du t de Student.

\section{RESULTATS}

L'âge moyen de la population étudiée est de 56,9 ans $(S D=11,8)$; la population était composée de 91 hommes et 9 femmes. Aucune différence significative d'âge en fonction du sexe n'a été notée. Le nombre de dents initial est de 15,7 dents $(S D=7,6)$ et la dose totale d'irradiation moyenne de 60,8 Grays (SD = 8,6).

La compliance pour le port des gouttières de fluoration est reportée sur la Fig. 1. Parmi les 100 patients de la cohorte, 48 n'ont jamais porté

Figure 1 : Compliance des patients au port des gouttières de fluoration

Compliance with the wear of fluorisation trays

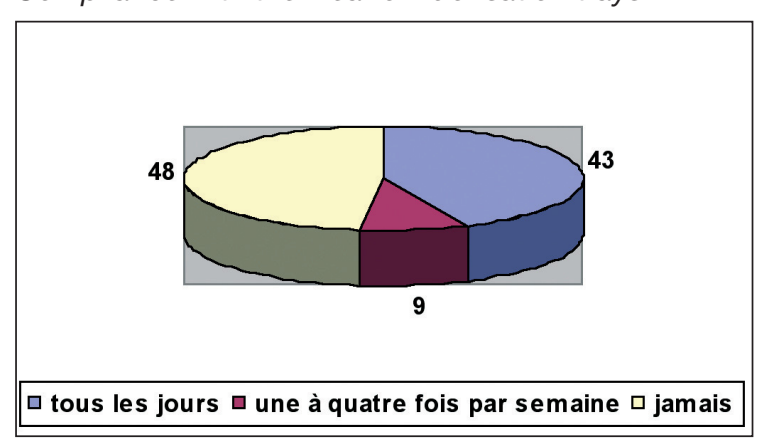

les gouttières et 43 ont respecté strictement le protocole de fluoration et porté leurs gouttières tous les jours. Neuf patients ont porté les gouttières occasionnellement (une à quatre fois par semaine). L'étude a donc montré un pourcentage élevé, et majoritaire, de patients ne respectant pas les conseils de fluoroprophylaxie. Aucune différence significative de la compliance en fonction de l'âge ou du sexe n'a été observée. Parmi les 48 patients qui n'ont jamais porté leurs gouttières de fluoration, 40 avaient des caries le jour de la consultation. Le nombre moyen des caries chez ces patients était de 6,1 (SD =5,2). Chez les 43 patients ayant strictement respecté le protocole et porté les gouttières, 12 présentaient des caries, avec un nombre de caries moyen de 5,1 $(S D=5,7)$. Cinq patients parmi les neuf qui portaient occasionnellement les gouttières avaient des caries lors de la consultation avec un nombre moyen de caries à 5,2 (SD=4). L'incidence de la compliance pour le port des gouttières de fluoration sur la carie dentaire est illustrée par la Fig. 2. L'augmentation de l'incidence de la carie est liée de façon significative au non-port des gouttières de fluoration $\left(\mathrm{Chi}^{2}, \mathrm{P}<10^{-6}\right)$. De plus, le nombre moyen de caries augmente de manière significative avec le degré de non-compliance (analyse de la variance, $p=0,0004)$.

Figure 2 : Diagramme comparatif compliance / carie dentaire

Compliance/carie comparative diagram

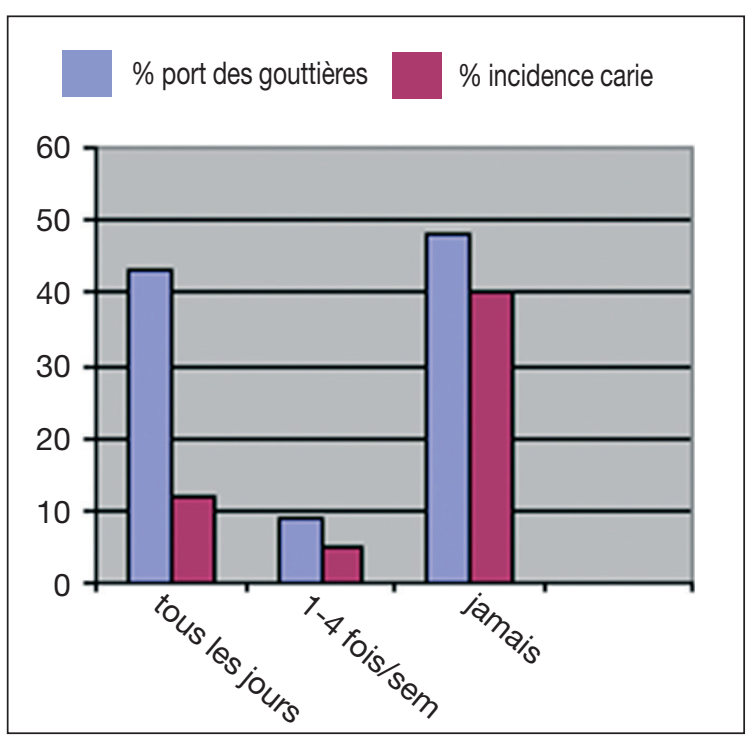

médecine buccale chirurgie buccale

VOL. $13, \mathrm{~N}^{\circ} 2$ 2007

page 79 
médecine buccale chirurgie buccale

VOL. $13, \mathrm{~N}^{\circ} 2$ 2007

page 80

\section{DISCUSSION}

La corrélation entre le non-port des gouttières de fluoration et l'apparition de caries dentaires montre une nouvelle fois le rôle majeur des fluorures dans la prévention des caries postradiques. La fluorothérapie topique à l'aide de gouttières fait partie intégrante de la prévention de ces caries [12,13]. Cependant, l'étude montre que plus de la moitié des patients n'a pas respecté le protocole de prophylaxie. Les patients compliants, respectant strictement le protocole, ne représentent que $43 \%$ des patients. Ces résultats amènent à s'interroger sur les raisons de ce faible pourcentage de port régulier des gouttières de fluoration chez les patients irradiés.

La plupart des cancers des VADS sont liés à une intoxication alcoolo-tabagique chronique, qui est souvent associée à une faible compliance pour les mesures d'hygiène buccale ${ }^{[7]}$. De plus, l'hyposialie perturbe les fonctions telles que la mastication, la gustation, la déglutition ou la phonation, ce qui altère de façon notable la qualité de vie des patients qui en souffrent. La qualité de vie dans les cancers des VADS est le sujet d'un nombre croissant d'études puisque la région cervico-faciale est le siège de fonctions majeures comme la communication, la nutrition ou la respiration. Les cancers dans cette région sont physiquement et émotionnellement dévastateurs. De plus, le manque de prévention des complications buccales liées à la radiothérapie et à l'hyposialie entraîne des risques accrus de lésions buccodentaires. Ces lésions peuvent avoir des conséquences sur la thérapie anti-cancéreuse, ou entraîner des infections nécessitant des chirurgies invasives qui introduisent une morbidité additionnelle et diminuent encore une fois la qualité de vie du patient [14]. Le contexte socio-économique du Nord Pas-de-Calais donne également des éléments d'explication au manque de compliance des patients. Cette région souffre d'un taux élevé de chômage, ce qui entraîne un stress supplémentaire et bien souvent l'augmentation des addictions telles que le tabac et l'alcool [15].
Dans ce contexte, la sensibilisation des patients aux mesures de prévention et d'hygiène buccale est des plus difficile.

L'expérience clinique révèle souvent :

- le manque ou l'absence de motivation du patient,

- une intolérance au port des gouttières due à des brûlures de la muqueuse buccale par le gel fluoré, - des gouttières mal ajustées qui entraînent des blessures de la muqueuse.

Ces observations rappellent le rôle majeur de toute l'équipe médicale qui prend en charge les patients irradiés. Plus spécifiquement, le chirurgien dentiste a un devoir d'information sur les risques liés au non-port des gouttières de fluoration. La compliance du patient sera basée sur la bonne compréhension des complications postradiques et du traitement préventif à vie que ces complications imposent. Le chirurgien dentiste doit bien entendu assurer le suivi à long terme de la fluorothérapie et remplacer les gouttières dès que cela est nécessaire. La communication entre le patient et le praticien est d'une importance capitale, et le rôle des professionnels de santé ne doit pas être limité aux actes cliniques. Les interactions sociales, un contact respectueux et chaleureux malgré les contraintes liées au temps, sont des éléments essentiels pour la compliance du patient [16].

Dans l'optique de réduire la morbidité et la mortalité liées aux cancers buccaux, des efforts de prévention primaire et secondaire sont nécessaires. Lors des visites régulières de contrôle, le chirurgien dentiste, en tant que professionnel de santé, doit inciter le patient à l'arrêt du tabac et de la consommation excessive d'alcool. Malheureusement, ces efforts de prévention primaire ne sont pas très répandus au sein des cabinets dentaires [17]. Le chirurgien dentiste est à la première place, lors des visites de contrôle, pour détecter une éventuelle récidive tumorale. Un examen rigoureux de la cavité buccale doit être réalisé pour le dépistage du cancer non seulement chez les patients irradiés mais aussi chez tous les patients présentant des facteurs de risque. 


\section{RÉFÉRENCES}

1 - Pasquesoone X, Chevalier D. Cancers des voies aérodigestives supérieures : le triste record français. Rev Prat $1999 ; 13: 213-5$.

2 - Aupérin A, Hill C. Epidémiologie des carcinomes des voies aérodigestives supérieures. Cancer Radiother $2005 ; 9: 1-7$.

3 - Liu RP, Fleming TJ, Toth BB, Keene HJ. Salivary flow rates in patients with head and neck cancer 0.5 to 25 years after radiotherapy. Oral Surg Oral Med Oral Pathol $1990 ; 70: 724-9$.

4 - Brown LR, Dreizen S, Handler S, Johnston DA. Effect of radiation-induced xerostomia on human oral microflora. J Dent Res 1975 ; 54 : 740-50.

5 - Cowman RA, Baron SS, Glassman AH, Davis ME, Strosberg AM. Changes in protein composition of saliva from radiation-induced xerostomia patients and its effects on growth of oral Streptococci. J Dent Res $1983 ; 62$ : 336-40.

6 - Garg AK, Malo M. Manifestations and treatment of xerostomia and associated oral effects secondary to head and neck radiation therapy. J Am Dent Assoc 1997 ; $128: 1128-33$.

7 - Epstein JB, Chin EA, Jacobson JJ, Rishiraj B, Le N. The relationships among fluoride, cariogenic oral flora, and salivary flow rate during radiation therapy. Oral Surg Oral Med Oral Pathol Oral Radiol Endod 1998 ; 86 : 286-92.

8 - Vissink A, Jansma J, Spijkervet FKL, Burlage FR, Coppes RP. Oral sequelae of head and neck radiotherapy. Crit Rev Oral Biol Med 2003 ; 14 : 199-212.

9 - Dreizen S, Brown LR, Handler S, Levy BM. Radiationinduced xerostomia in cancer patients. Effect on salivary and serum electrolytes. Cancer 1976 ; 38 : 273-8.
10 - Jenkins GN. Theories on the mode of action of fluoride in reducing dental decay. J Dent Res 1963 ; 2 : 44452.

11 - Epstein JB, Van der Meij EH, Lunn R, StevensonMoore P. Effects of compliance with fluoride gel application on caries and caries risk in patients after radiation therapy for head and neck cancer. Oral Surg Oral Med Oral Pathol Oral Radiol Endod 1996 ; 82 : 268-75.

12 - Horiot JC, Malka G, Schraub S, Le Dorze C, Ramadier J, Martin P, Jampolis S. Prévention des caries et des ostéonécroses par fluoruration quotidienne locale chez les malades irradiés. Rev Stomatol 1977 ; 78 : 385-96.

13 - Vérain A. La florothérapie post-radique. Rev Stomatol Chir Maxillofac 2004 ; 105 : 289-90.

14 - Lockhart PB, Clark J. Pretherapy dental status of patients with malignant conditions of the head and neck. Oral Surg Oral Med Oral Pathol 1994 ; 77 : 236-41.

15 - Greenwood M, Thomson PJ, Lowry RJ, Steen IN. Oral cancer: material deprivation, unemployment and risk factor behaviour - an initial study. Int J Oral Maxillofac Surg $2003 ; 32: 74-7$.

16 - Gamble K. Communication and information: the experience of radiotherapy patients. Eur $\mathrm{J}$ Cancer Care $1998 ; 7:$ : 153-61.

17 - Cruz GD, Ostroff JS, Kumar JV, Gajendra S. Preventing and detecting oral cancer: oral health care providers'readiness to provide health behavior counseling and oral cancer examinations. J Am Dent Assoc 2005 ; $136:$ 594-601.

\section{médecine buccale chirurgie buccale}

VOL. $13, \mathrm{~N}^{\circ} 2$ 2007

page 81 